\title{
Accumulation of prion protein in tonsil and appendix: review of tissue samples
}

\author{
David A Hilton, Azra C Ghani, Lisa Conyers, Philip Edwards, Linda McCardle, Mark Penney, \\ Diane Ritchie, James W Ironside
}

Variant Creutzfeldt-Jakob disease is almost certainly caused by the bovine spongiform encephalopathy agent, and although the disease is rare (115 deaths to date) there is uncertainty about future numbers of cases. The lack of a conventional immune response and the inability to detect abnormal prion protein in blood has hampered the development of a blood test. ${ }^{1}$ Lymphoreticular accumulation of prion protein has been used as a preclinical test for scrapie (the form of the disease in sheep and goats) and is a consistent feature of variant Creutzfeldt-Jakob disease, occurring before the onset of symptoms. ${ }^{2-4}$ We screened large numbers of specimens from appendicectomies and tonsillectomies for the presence of prion protein in lymphoreticular tissue to determine the number of people with preclinical variant Creutzfeldt-Jakob disease.

\section{Methods and results}

We retrieved appendix and tonsil samples removed between 1995 and 1999 from patients aged 10-50 from histopathology departments across the United Kingdom. The samples were anonymised before testing. We also examined appendix samples removed at autopsy or at surgery from patients with variant Creutzfeldt-Jakob disease. Prion protein was detected by immunohistochemistry with monoclonal antibodies 3F4 (Dako, Ely, Cambridge) and KG9 (Institute for Animal Health, Newbury) and visualised with the catalysed signal amplification system (Dako). We excluded cases with fewer than five secondary lymphoid follicles from the final analyses because in the previously reported case and those examined at autopsy, prion protein was present in around $20 \%$ of follicles. ${ }^{4}$ We tested 11228 samples, of which 2910 were excluded. Most samples were appendixes and $70 \%$ were from patients aged 20-29. In one case we identified prion protein immunoreactivity in a single lymphoid follicle stained with KG9 (figure). The pattern of staining was similar to that seen in the two other patients who developed variant CreutfeldtJakob disease (figure $\left.{ }^{1}\right){ }^{4}$ This positive staining was less evident in sections stained with $3 \mathrm{~F} 4$ antibody. The reason for this discrepancy is not clear but may be due to sampling error because of the focal nature of prion protein deposition. In the appendixes removed before the onset of symptoms in three patients with variant Creutfeldt-Jakob disease, two (removed in 1995 and 1996; see figure) were positive, and the third (removed in 1987) was negative. ${ }^{4}$ Overall, 19 of 20 appendixes removed at autopsy from cases of variant CreutzfeldtJakob disease with adequate numbers of lymphoid follicles had lymphoreticular accumulation of prion protein.

\section{Comment}

One appendix showing the lymphoreticular accumulation of prion protein out of 8318 samples tested gives an estimated detectable prevalence of prion protein accumulation of 120 per million $(95 \%$ confidence interval, 0.5 to 900 ) among people aged $10-50$ between 1995 and 1999. Our study design precluded transmission studies, but the accumulation of prion protein in lymphoreticular tissue detected by the immunohistochemical technique correlates with the detection of abnormal prion protein by western blotting and remains the only technique shown to reliably predict disease in animals. ${ }^{2}{ }^{35}$ The sensitivity and specificity of preclinical lymphoreticular accumulation of prion protein in predicting variant Creutzfeldt-Jakob disease are unknown, but in sheep exposed to scrapie are estimated to be $87 \%$ and $94 \%$, respectively. ${ }^{5}$ At autopsy, $95 \%$ of appendixes from patients with variant Creutzfeldt-Jakob disease with adequate amounts of lymphoid tissue showed accumulation of prion protein, as did both appendixes removed during the 1990s from patients who later developed the disease.

We provide the first estimate of the number of people who may be a potential source of variant Creutzfeldt-Jakob disease by iatrogenic spread. Large scale prospective screening of tissue from tonsillectomies is needed to obtain precise data on prevalence. The use of fresh tissue would allow for a semiautomated western blot assay and verification of positive samples by transmission studies. As half of tonsillectomies are in children under 10, who will have had little or no exposure to bovine spongiform encephalopathy, the opportunity for such a study will diminish over time.

We thank histopathology departments and relatives of patients with variant Creutzfeldt-Jakob disease who gave consent for autopsy tissues to be used as positive control material.

Contributors: DAH designed the English study, screened samples tested at Plymouth, and drafted the manuscript; he will act as guarantor for the paper. JWI designed the Scottish study, screened samples tested at Edinburgh, and contributed to the manuscript. ACG performed the statistical analyses and contributed to the manuscript. MP, LC, and PE performed the technical work at Plymouth. LMcC and DR performed the technical work and assisted in screening samples at Edinburgh.
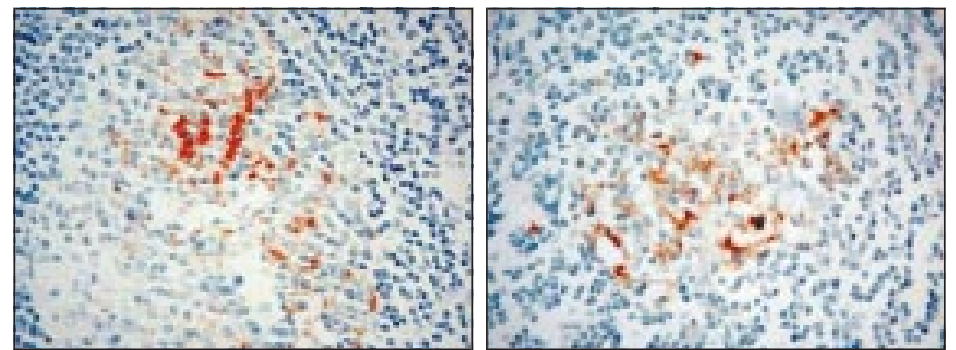

(Left) Distribution of coarse granular prion protein within lymphoid follicle of appendix, suggestive of follicular dendritic cells. (Right) Lymphoreticular accumulation of prion protein in appendix tissue from patient with Creutzfeldt-Jakob disease, removed two years before onset of symptoms

\author{
Department of \\ Histopathology, \\ Derriford Hospital, \\ Plymouth PL6 8DH \\ David A Hilton \\ neuropathologist \\ Lisa Conyers \\ research technician \\ Philip Edwards \\ biomedical scientist \\ Mark Penney \\ research technician \\ Department of \\ Infectious Disease \\ Epidemiology, \\ Faculty of Medicine, \\ Imperial College of \\ Science, Technology \\ and Medicine, \\ London SW7 2AZ \\ Azra C Ghani \\ research fellow \\ National CJI \\ Surveillance Unit, \\ University of \\ Edinburgh, \\ Edinburgh \\ EH4 2XU \\ Linda McCardle \\ biomedical scientist \\ Diane Ritchie \\ research assistant \\ James W Ironside \\ professor of clinical \\ neuropathology \\ Correspondence to: \\ D A Hilton \\ david.hilton@ \\ phnt.swest.nhs.uk
}

BMJ 2002;325:633-4 
Funding: Department of Health and the Royal Society. The views expressed in this publication are those of the authors and not necessarily those of the Department of Health.

Competing interests: None declared.

1 Collinge J. Variant Creutzfeldt-Jakob disease. Lancet 1999;354:317-23

2 Schreuder BEC, van Keulen LJM, Vromans MEW, Langeveld JPM, Smits MA. Preclinical test for prion diseases. Nature 1996;381:563.
3 Hill AF, Butterworth RJ, Joiner S, Jackson G, Rosser MN, Thomas DJ, et al Investigation of variant Creutzfeldt-Jakob disease and other human prion disease with tonsil biopsy samples. Lancet 1999;353:183-9.

4 Hilton DA, Fathers E, Edwards P, Ironside JW, Zajicek J. Prion immunoreactivity in appendix before clinical onset of variant Creutzfeldt-Jakob disease Lancet 1998;352:703-4.

5 O'Rourke KI, Baszler TV, Besser TE, Miller JM, Cutlip RC, Wells GAH, et al. Preclinical diagnosis of scrapie by immunohistochemistry of third eyelid lymphoid tissue. J Clin Microbiol 2000;38:3254-9. (Accepted 30 July 2002) p 648

Department of Public Health and Clinical Medicine,

Umeå University Hospital, Umeå, S-901 85, Sweden Birgitta Stegmayr associate professor Kjell Asplund professor

Correspondence to: B Stegmayr birgitta.stegmayr@ medicin.umu.se

BMJ 2002;325:634-5
Education and debate

\section{Informed consent for genetic research on blood stored for more than a decade: a population based study}

\author{
Birgitta Stegmayr, Kjell Asplund
}

With recent advances in molecular genetics, there has been a surge in interest in using stored blood samples for genetic research, even though informed consent at the time of blood sampling did not include this possibility. One of the cornerstones of the World Medical Association's Declaration of Helsinki on ethical principles for medical research is the need for informed consent and the right of any participant in a research project to withdraw at any time. ${ }^{1}$ We report here our experiences of seeking informed consent for academic and commercial genetic research on blood samples collected more than a decade earlier.

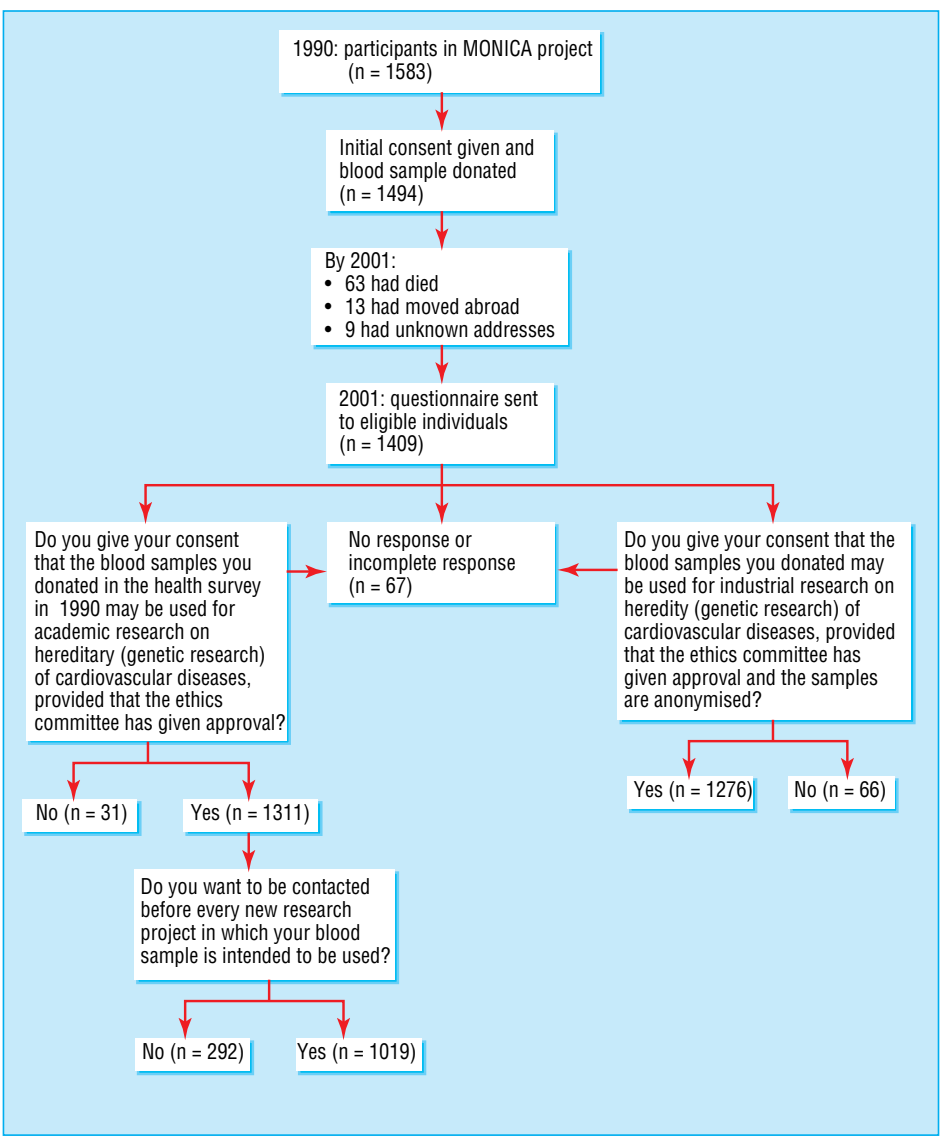

Follow up in 2001 of participants in 1990 MONICA survey, showing numbers of participants lost to follow up and responses to questionnaire about consent for blood to be used in genetic research

\section{Methods and results}

A total of 1583 out of 2000 (79.2\%) randomly selected men and women in the age group 25-64 years participated in the 1990 risk factor survey in the World Health Organization's MONICA project. ${ }^{2}$ Participants were given written information and asked to donate blood for "future research on cardiovascular disorders and diabetes." A total of 1494 blood samples were taken (in 89 participants, consent was not given or technical problems arose in obtaining a blood sample). The blood samples were fractionated and stored at $-80^{\circ} \mathrm{C}$.

In 2001, 11 years later, 85 of the 1494 individuals had died, moved abroad, or had an unknown address (figure). We sent a letter to the remaining 1409 participants with information about ongoing genetic studies and seeking consent at three different levels (figure). Of the 1409 subjects, 1342 (95.2\%) responded.

A total of 1311 out of 1409 (93.0\%) eligible participants gave their consent for their blood samples to be used for academic genetic research, provided that the ethics committee had approved the research. Thirty one (2.2\%) participants did not give their consent; 64 participants did not reply and three provided incomplete answers (4.8\% together).

Of the 1311 participants who gave their consent, $292(22.3 \%)$ wanted to be informed about, and give new consent for, each new genetic project (figure). The rest gave general consent to genetic research, as long as an ethics committee had approved the research. However, a further $35(2.5 \%)$ participants did not give consent for their blood to be used for industrial genetic research (figure).

\section{Comment}

Eleven years after donation of samples, only a very small proportion of participants did not give consent for their blood to be used in academic or industrial genetic research.

To our knowledge, this report is the first to provide empirical data from a "real life" situation-that is, people's willingness to give consent to genetic research on their own blood donated more than a decade previously, when genetic research was not an issue. Participation in risk factor screening is, in itself, an expression of willingness to contribute to research. The participants in the original survey in 1990 were randomly selected, with a participation rate as high as $79 \%$, thus suggesting that 